\title{
Zur Anziehungskraft eines Gewaltvideos
}

Am 15. März 2019 konnte potenziell die ganze Welt gleichsam in Echtzeit zusehen, wie sich ein terroristischer Massenmord ereignete. Als sich zahlreiche Gläubige zum Freitagsgebet in einer Moschee im neuseeländischen Christchurch versammelt hatten, stürmte ein rechtsextremer Täter das Gotteshaus, filmte sein Verbrechen und streamte es live auf Facebook - fast 17 Minuten lang konnte er „Drehbuchautor [und] Regisseur [...] seines eigenen Films sein “. Als der Stream abgebrochen wurde, war der Täter - wie sich im Zuge der Ermittlungen später herausstellte - unterwegs zu einer weiteren Moschee. Bei dem Anschlag starben an insgesamt zwei Tatorten 51 Menschen; 50 weitere wurden verletzt.

\section{Was zu zeigen sein wird}

Mit der kriminell eingesetzten Wechselbeziehung von physischem Gewaltakt und technischen Aufzeichnungs- und Verbreitungsmitteln markiert die Tat einen Verbrechenstypus, der in digital vernetzten Bild- und Bildnutzungskulturen globale Wirkung entfalten kann: das terroristische Aufmerksamkeitsverbrechen. ${ }^{2}$ Und damit ordnet sich die Tat auch in eine kontrovers geführte Mediendebatte über Terrorbilder ein, die immer wieder fallspezifisch an Intensität gewinnt: Unter welchen Bedingungen können, dürfen oder müssen sie gezeigt bzw. nicht gezeigt werden? ${ }^{3}$ In der Berichterstattung über das Video von Christchurch wurden primär medienethische und -politische Gesichtspunkte diskutiert. Dabei markierten gerade jene Redaktionen, die sich für eine Bildpublikation entschieden, implizit aber auch die Bedeutung von bildkritischen Analysen: Woher rührt die Anziehungskraft eines potenziell abstoßenden Terrorvideos?

1 Arno Frank: Wir schauen nicht weg, 15.03.19, Der Spiegel, https://www.spiegel.de/kultur/gesellschaft/ christchurch-und-das-internet-das-gestreamte-massaker-a-1258167.html (Stand 3/2020).

2 Die Verbindung von Terror- und Mediengewalt wird vielfach als Symbiose beschrieben. Vgl. exemplarisch Muhammad Ayish: ,Employing of Media during Terrorism’. In: Mahmoud Eid (Hg.): Exchanging Terrorism Oxygen for Media Airwaves. The Age of Terroredia, Hershey 2014, S. 157172; zum Begriff des Aufmerksamkeitsverbrechens vgl. Philipp Müller: Realitätenkollaps? Zum Status von Bild und Betrachter bei Gewaltvideos. In: Karen Fromm, Sophia Greiff, Anna Stemmler (Hg.): Images in Conflict, Weimar 2018, S. 80-102, hier: S. 87f.; Christchurch war indes nicht das erste live gestreamte Aufmerksamkeitsverbrechen. Vgl. Fridtjof Küchemann: Eine Plattform für Verbrecher, 15.06.16, FAZ, https://www.faz.net/aktuell/feuilleton/medien/facebook-live-eine-plattformfuer-verbrecher-14289020.html (Stand 4/2020); vgl. Lindsey Bever u. a.: ,Facebook killer ' dies after three-day police pursuit in Pennsylvania, 18.04.17, Washington Post, https:/www.washingtonpost. com/news/post-nation/wp/2017/04/18/facebook-murder-suspect-steve-stephens-is-dead-police-say/ (Stand 8/2020).

3 Vgl. Franca Buss, Philipp Müller: Hin- und Wegsehen. Formen und Kräfte von Gewaltbildern. In: dies. (Hg.): Hin- und Wegsehen. Formen und Kräfte von Gewaltbildern, Berlin/Boston 2020, S. 11-45. 
In Auseinandersetzung mit Videoausschnitten und -stills, die die Bildzeitung publizierte, rücken in diesem Aufsatz die Bilder selbst und deren Wirkungskräfte als nicht zu unterschätzende Teile bestimmter Wahrnehmungsmuster und Nutzungsbedürfnisse ins Zentrum. Anhand der an Ego-Shooter-Spiele erinnernden Ästhetik des Terrorvideos und funktionsbestimmender Spezifika von Body-Cams als „Nahkörpertechnologien“ wird - unter kritischer Berücksichtigung ideologischer und ethischer Problemstellungen - die immersive Einbindung der Betrachter*innen aus bild- und affekttheoretischer Perspektive untersucht. ${ }^{4}$ Ausblickend werden sich die Bedingungen der Zeigbarkeit zu aktuellen Zensurfragen um gesetzliche Löschungs- und Filterungsvorgaben zuspitzen.

In den ersten 24 Stunden konnte Facebook das Video zwar millionenfach entfernen, doch war es trotzdem untilgbar geworden. Hierfür tragen aber nicht nur Plattformen, sondern auch deren Nutzer*innen Verantwortung. ${ }^{5}$ Ebenso wie Rezeptionsakte die kommunikative Reichweite der Tat vergrößern kann sich im Hybrid von Massenmedien und sozialen Netzwerken schon die Bild(mit)teilung einer einzigen Person auf den Reiz- und Informationshaushalt großer Gruppen auswirken. Dies hatte sich auch der Täter zunutze gemacht. ${ }^{6}$ So werden Nutzungsbedürfnisse als integrale Teile der Sichtbarwerdung von Gewaltbildern erklärungsbedürftig. Ebenso wie Redaktionen und Nutzer*innen sind auch Wissenschaftler*innen zur Selbstreflexion aufgerufen. Hinsichtlich der Frage, was Leser*innen visuell zugemutet werden kann, wird dieser Text keine bilderlose Bilduntersuchung bleiben; ${ }^{7}$ doch bedeutet das Zeigen unbedingt „keine Aufforderung zum Ansehen jedweder Gräuelbilder“ oder die Grundlegung einer Bildnegierungspauschale, sondern dient der differenzierten Überprüfung beider Zeigeoptionen. ${ }^{8}$

Sobald das Teilen und Ansehen von Tat- oder Täterbildern die Intention des Aufmerksamkeitsverbrechers erfüllen und sich Publikations- oder Nutzungsverhalten nicht kritisch von dessen Inszenierungslogik absetzen, stellt sich die Frage: „Hat er uns

4 Timo Kaerlein: Smartphones als digitale Nahkörpertechnologien: zur Kybernetisierung des Alltags, Paderborn 2018.

5 Vgl. Charlotte Klonk: Terror. Wenn Bilder zu Waffen werden, Frankfurt a. M. 2017, S. 92.

6 Vgl. Bernhard Pörksen: Die große Gereiztheit. Wege aus der kollektiven Erregung, München 2018, S. 12 und 88 .

7 Vgl. Marion Müller: Burned Bodies. Visueller Horror als strategisches Element kriegerischen Terrors eine ikonologische Betrachtung ohne Bilder. In: dies., Thomas Knieper (Hg.): War Visions, Köln 2005, S. 405-423; vgl. Müller (s. Anm. 2).

8 Linda Hentschel: Gewaltbilder und Schlagephantasien oder: Die Rebellion der Betrachtermelancholie. In: Julia Bee u. a. (Hg.): Folterbilder und -narrationen. Verhältnisse zwischen Fiktion und Wirklichkeit, Göttingen 2013, S. 115-127, hier: S. 118. 
alle zu Komplizen gemacht? "99 Durch Live-Funktionen sozialer Plattformen verschärft sich die Frage nach der Mittäterschaft, die regelmäßig die Terrorberichterstattung mitbestimmt. Global vernetzte digitale Bildkulturen können zwar durchaus (bürger-) journalistische Produktivkräfte freisetzen. ${ }^{10}$ Doch sie bieten ebenso Verbrechern attraktive Inszenierungsoptionen: „Jedem medientechnischen Durchbruch folgt eine neue Ausprägung des Terrorismus." ${ }^{11}$

In Konkurrenz zu anderen Augenzeug*innen mit internetfähigem Smartphone oder zu Redaktionen, deren Berichterstattung sich den Hochgeschwindigkeitsdynamiken sozialer Netzwerke anzupassen versucht, kann wiederum ein Publikationsdruck entstehen, der ethische Reflexionen sekundarisiert. ${ }^{12}$ Gerade Live-Berichte in Terrorkontexten erregen oft den Verdacht kalkulierter oder unbedachter Sensationalisierung, wenn beängstigende Bilder die Welt teils noch vor polizeilichen Statements zur Sachlage erreichen. Bildliche Affekt- und Informationspotenziale drohen zusammenzufallen. Die Live-Funktion als zeitliche Kompression von Produktion, Rezeption und Distribution lässt zudem Betrachter*innen kaum Raum zur Reaktion und kann jene affektiven Unmittelbarkeitseffekte verstärken, die vor allem technische Gewaltbilder durch ihre mimetischen und indexikalischen Qualitäten besitzen - obgleich sie durch postproduktive Manipulierbarkeit auch zu Skepsis an ihrem Wirklichkeitspotenzial oder zu Verschwörungsmythen verleiten. ${ }^{13}$

\section{Zeigen und Nicht-Zeigen}

In der Diskussion um das Video von Christchurch plädierten die meisten Redaktionen schriftlich für eine Bildnegierung, um dem Täter die Deutungshoheit zu nehmen. Zwangsläufig mussten sie dabei dem Dilemma visueller Negation von Aufmerksam-

9 Moritz Aisslinger, Bastian Berbner, Alice Bota u. a.: Hier gibt es nichts zu sehen, in: Die ZEIT, am 21.03.2019, S. 13-15. Ein historischer Debatteneinblick bei Buss, Müller (s. Anm. 3), S. 13-15; zu Christchurch vgl. Lorenz Meyer: Christchurch, Künstlerische Freiheit, Tragödienjournalismus, bildblog.de, $18.03 .19(3 / 20)$.

10 Vgl. Kerstin Schankweiler: Bildproteste. Widerstand im Netz, Berlin 2019; vgl. Julia Höner, Kerstin Schankweiler (Hg.): Affect Me. Social Media Images in Art, Ausst.kat., Leipzig 2017.

11 Bastian Berbner: Wir Terrorhelfer, 23.08.17, Zeit Online, https://www.zeit.de/2017/35/journalismusterrorismus-anschlaege-medien-bilder-umgang (Stand 3/2019).

12 Zur „Geschwindigkeitsfalle“ der Gewaltberichterstattung vgl. Bastian Berbner, Franca Buss, Philipp Müller: Wir Terrorhelfer? In: Buss, Müller (s. Anm. 3), S. 311-325, hier S. 316.

13 Das Technische meint hier Fotos und Videos mit operativem Potenzial in der affektorientierten Berichterstattung. Zur semantischen und historischen Erweiterung vgl. Angela Fischel: Bildbefragungen. Technische Bilder und kunsthistorische Begriffe. In: Horst Bredekamp, Vera Dünkel, Birgit Schneider (Hg.): Das technische Bild. Kompendium zu einer Stilgeschichte wissenschaftlicher Bilder, Berlin 2008, S. 14-24, hier: S. 14. 

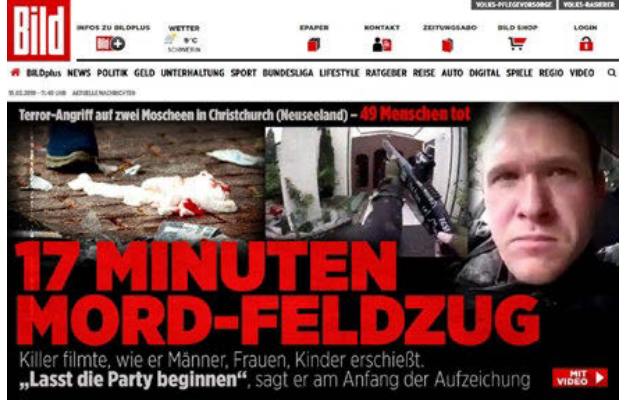

1: Bild.de: Startseite vom 15. März 2019 um 11:40 Uhr.

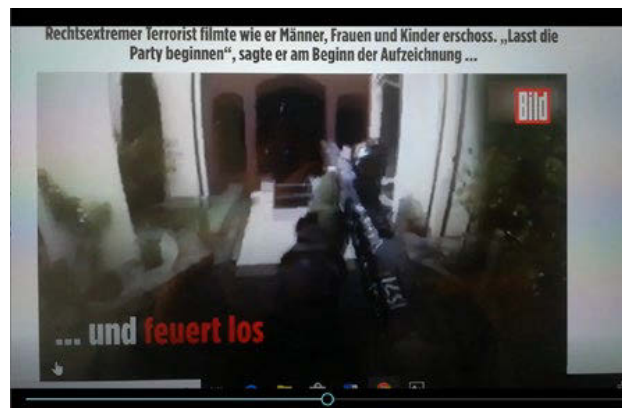

2: Bild.de: 17 Minuten Mordfeldzug, 15. März 2019, Video, 2:18 min, TC: 01:08.

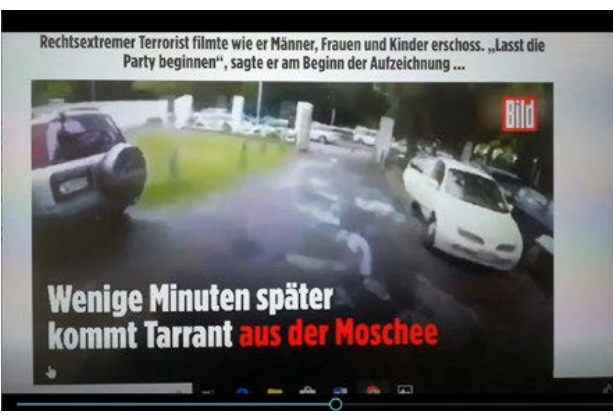

3: Bild.de: 17 Minuten Mordfeldzug, 15. März 2019, Video, 2:18 min, TC: 01:12. keitsverbrechen zwischen Fremd- und Selbstzensur aufsitzen. Wenige Redaktionen, wie etwa Die Zeit und die Hamburger Morgenpost, hoben sich hervor, indem sie das Nicht-Zeigen als Figur der Negation visualisierten und ein weiteres Dilemma markierten. Die Bildverweigerung als Bild stiftet auch jene Aufmerksamkeit für den Täter, die sie ihm als damnatio memoriae nehmen möchte und kann sich medienethisch verkehren, wenn sie dramatisierende Vorstellungen des Monströsen erzeugt oder dazu motiviert, die Bilder selbst zu suchen. ${ }^{14}$

Um 17.03 Uhr des Tattages veröffentlichte bild.de den Artikel „17 Minuten Mordfeldzug“ und bewarb ihn auf der Startseite explizit mit Täterselbstbild und „MIT VIDEO“. х Abb. 1 Im Untertitel vibrierten Worte des Täters ganz im Sinne von dessen Aufmerksamkeitsstrategie: „,Lasst die Party beginnen', sagte er am Beginn der Aufzeichnung ..."15

Der etwa 2-minütige Clip auf bild.de führt mitten ins Geschehen. In Ego-Perspektive holt der Täter eine Waffe aus dem Kofferraum und geht auf die Moschee zu. Schnitt. Rückblende. Der Täter zeigt sich im Auto: „Let's get this party started.“ Schnitt. Am Eingang der Moschee kommt - wieder in Ego-Perspektive - der Lauf eines Gewehrs in Bild- und Blickfeld. $\boldsymbol{x}$ Abb. 2 Schnitt. Der Täter geht zum Auto, schießt auf dem Weg dorthin um sich, holt eine neue Waffe aus dem Kofferraum und kehrt zurück zur Moschee. $\pi$ Abb. 3 Schnitt. Der Täter sitzt mit

14 Vgl. Buss, Müller (s. Anm. 3), S. 18.

1517 Minuten Mordfeldzug, 15.03.19, Bild, https://www.bild. de/news/ausland/news-ausland/christchurch-neuseelandterrorist-filmte-anschlag-in-moscheen-live-60677536.bild. html (Stand 3/2019). 
Gewehr am Steuer. Während der Fahrt zum zweiten Tatort zerschießt er die Scheibe des Beifahrersitzes. Als versuchte die Bildzeitung die Tatbilder wirkungsstrategisch auszugleichen, beendet sie den Beitrag mit einem Handyvideo, das aus einem fahrenden Auto heraus zeigt, wie Polizisten einen Mann zu Boden drücken - ob es sich um den Täter handelt, ist unklar.

Wochen später folgte eine Rüge des Presserats: die Bildzeitung zeige ,nicht die Taten selbst“, bediene aber „überwiegend Sensationsinteressen“; ; ${ }^{16}$ der Clip blieb online. Da Gewalttat und deren Medialisierung intentional verbunden sind, müsste allerdings jeder Ausschnitt als Tatbild gelten. Auch die Ausschnitthaftigkeit des Clips bzw. die spezifische Anordnung der redaktionell gewählten Ausschnitte mit dramatisierend hohem Schnitttempo ist problematisch. Denken wir „in Szenarien“, wie sie uns ,alltäglich im Entwerfen von Handlungsabläufen [...] begegnen“, so erzeugt der Clip keinen reflexiven Abstand, sondern Imaginationen des Mordens und Sterbens in der Moschee. ${ }^{17}$ Er rückt nah und kann Ängste vor Gewalt an vergleichbaren öffentlichen Orten im eigenen Alltag wecken.

Ein Jahr nach Publikation des Artikels scheinen Text und Video bearbeitet worden zu sein. Nach einer Videobeschreibung bis zum ersten Schnitt vor dem ersten Schuss ist zu lesen: „Ab hier zeigt BILD [...] nur noch Standbilder. Die Bewegtbild-Szenen sind unerträglich. ${ }^{{ }^{118}}$ In der Version vom 15.03.19 aber gab es keine Standbilder. ${ }^{19}$ Ein redaktioneller Hinweis fehlt. ${ }^{20}$ Die Schnitte der ersten Version füllen nun Standbilder des Mordens, die gleichsam die vormals erzeugten Gewaltimaginationen einholen. Verstärkt werden diese zudem durch ekphrastische Schilderungen des Tathergangs in knappen Schlagsätzen, die mit den Standbildern eingeblendet werden. Schon am 16.03.19 veröffentlichte die Bildzeitung auf dem Titelblatt der Printausgabe das Tätergesicht und ein Tatbild mit Waffe aus dem Video sowie ein Leichenfoto aus anderer Quelle. Im

16 Redaktion hat sich zum Werkzeug des Täters gemacht, 07.06.19, presserat, https://www.presserat.de/ presse-nachrichten-details/christchurch-video-redaktion-hat-sich-zum-täter-werkzeug-gemacht.html (Stand 3/2020).

17 Andreas Wolfsteiner: Ungrund, Szenario. Appräsentation und Wahrnehmungsentzug. In: Markus Rautzenberg, Juliane Schiffers (Hg.): Ungründe. Potenziale prekärer Fundierung, Paderborn 2016, S. 115-127, hier: S. 117.

1817 Minuten Mordfeldzug (s. Anm. 15).

19 Auch die Rüge des Presserats scheint die Videoversion ohne Standbilder aus dem Inneren der Moschee (,die Taten selbst“) zu adressieren.

20 Wann Texthinweis und zweite Videoversion gepostet wurden, ist unklar. Auffällig ist, dass die Bildzeitung am 15.03.19 abends einen zweiten Artikel mit Ausschnitten aus dem Tätervideo veröffentlicht, das exakt die gleiche Länge hat wie die jetzige Version des ersten Artikels. Vgl. Julian Reichelt: Ein feiger, niederträchtiger Mörder, 15.03.19, Bild, https://www.bild.de/politik/kolumnen/kolumne/kommentarzu-christchurch-terror-ein-feiger-niedertraechtiger-moerder-60694360.bild.html (Stand 3/2019). 
Innenteil folgte ein visuelles „Protokoll des 17-Minuten-MASSAKERS“ mit weiteren Screenshots, die vergleichbar sind mit den Standbildern aus dem Online-Clip: Verletzte und Tote; am Boden liegende Menschengruppen, auf die geschossen wird. $\boldsymbol{x}$ Abb. 4

„Erst die Bilder“, so Julian Reichelt, der Vorsitzende der Chefredaktionen der Bildzeitung, demonstrierten „die erschütternde menschliche Dimension dieser Schreckenstat“. Journalismus sei dazu da, „Bilder der Propaganda und Selbstdarstellung zu entreißen und sie einzuordnen“. ${ }^{21}$ Doch steigert das visuelle Echo potenziell nicht eher die „Dimension“, indem es den Wirkungsradius der Bilder als intentionale Teile der Tat ebenso vergrößert wie sich die Texte dem Affektgrad des Videos anpassen und reflexive Distanzierung erschweren? Umgehend setzte Kritik an Reichelts Aussagen ein. ${ }^{22}$

Um auf die Gefahr der Bedürfnisbefriedigung nicht nur von naiven Schaulustigen, sondern auch von Sympathisant*innen und potenziellen Nachahmungstäter*innen hinzuweisen, sind ethische und politische Argumente gegen das Zeigen wichtig. In der Tat ereigneten sich 2019 weitere Gewaltakte, bei denen sich Täter oder Tatmuster auf den Terror von Christchurch bezogen. ${ }^{23}$ Doch ebenso können Personen einen starken Betrachtungsreiz verspüren, deren ethische Grundhaltung diesem entgegensteht. Damit ein Konflikt zwischen persönlichen Moralvorstellungen und Rezeptionsbedürfnissen, der durchaus zu Fremdheitsgefühlen gegenüber sich selbst führen kann, aber zu nutzungskritischer Selbstbefragung anregt, ist reflexive Distanz notwendig, weswegen im Folgenden eine bildkritische Analyse der Nähe erzeugenden Affizierungskräfte der technischen Gewaltbilder vorgeschlagen wird.

„Perfide“ sei, so Arno Frank, „dass sich die Bilder der Gewalt an den Primaten in uns richten. Der verängstige Affe muss der Gefahr ins Auge sehen. “24 Ohne diese diskutable evolutionspsychologische Einhüllung des komplexen Verhältnisses von Gewaltbildern und deren Betrachter*innen hier weiter zu verfolgen, umkreist Frank doch die entscheidende Kategorie, um den angeblichen Betrachtungszwang theoretisch einzuordnen: den Affekt.

21 Ebd.

22 Vgl. Boris Rosenkranz: Nichts gelernt: In der Journalistenschule mit Julian Reichelt, 18.03.19, Übermedien, https://uebermedien.de/36432/nichts-gelernt-in-der-journalistenschule-mit-julian-reichelt/ (Stand 3/2019); vgl. Robert Tusch: Unfassbar: Kritik an Bild-Chef Julian Reichelt, 16.03.19, MEEDIA, https://meedia.de/2019/03/16/unfassbar-kritik-an-bild-chef-julian-reichelt-wegen-veroeffentlichungvon-szenen-aus-live-video-des-attentaeters/ (Stand 1/2020).

23 Das investigative Recherchenetzwerk Bellingcat wies sofort auf mögliche Nachahmungstaten hin. Vgl. Robert Evans: Shitposting, Inspirational Terrorism, and the Christchurch Mosque Massacre, 15.03.19, Bellingcat, https://www.bellingcat.com/news/rest-of-world/2019/03/15/shitposting-inspirational-terrorismand-the-christchurch-mosque-massacre/ (Stand 1/2020). Vor allem der Terror in Halle am 09.10.10 wurde vielfach mit Christchurch verglichen.

24 Frank (s. Anm. 1). 


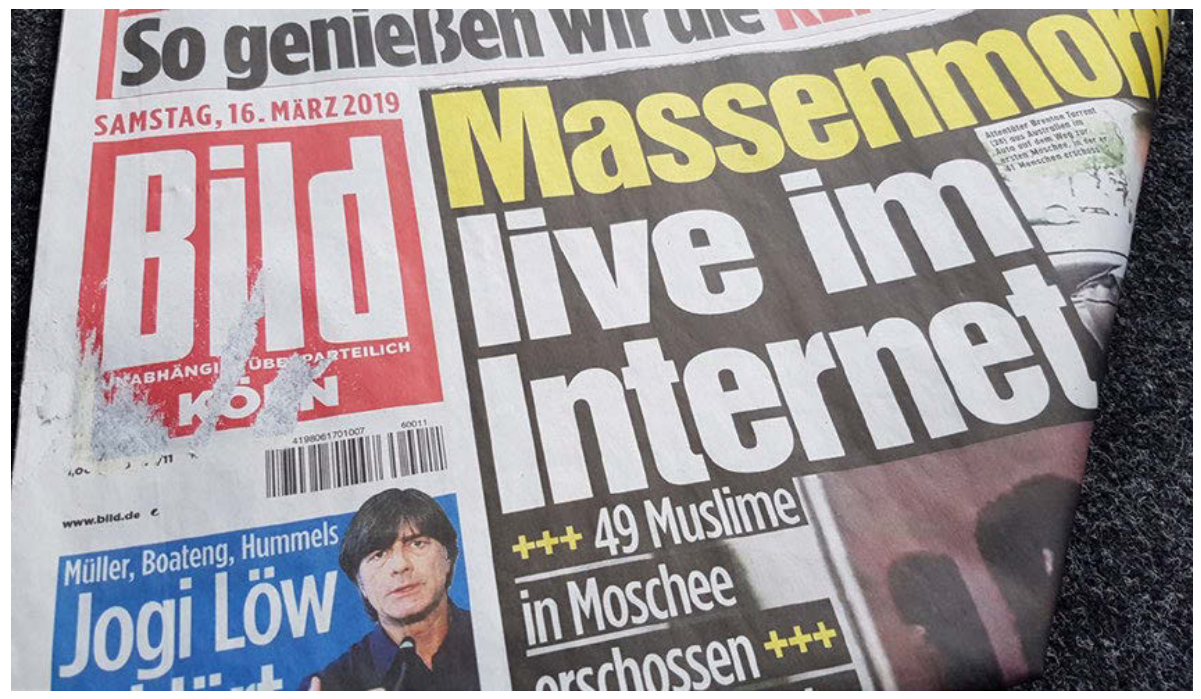

4: Titelseite der Bild vom 16. März 2019.

Oft als „kurze Reize“ beschrieben, verleiten Affekte vermeintlich „schneller als das Denken zu einer Aktion“ und erschweren den (Wieder-)Aufbau von reflexiver Distanz..$^{25}$ Gerade deswegen erscheinen technische Gewaltbilder wie jene aus Christchurch so „verführerisch in der affektiven Intensität“ wie ,verhängnisvoll in der angeblichen Beeinträchtigung des Reflexionsvermögens “. ${ }^{26}$ Doch sind wir als Nutzer*innen nicht restlos reflexionsohnmächtig gegenüber den eigenen Rezeptionsbedürfnissen, sodass vom Affekt nicht als „,rein vorreflexive Gefühlsregung“, sondern vielmehr von Affektdynamiken „,zwischen“ Körpern“ “27 zwischen Bildern und Nutzer*innen auszugehen ist. ${ }^{28}$ So richtet sich der Fokus sowohl auf die Subjekte und deren Rezeptionsmuster als auch auf die bildlichen Entstehungsgründe dieses affektiven Wechselwirkungsverhältnisses, sobald das Terrorvideo angeboten wird und Personen dem Betrachtungsreiz nachgehen, um es sich anzusehen.

25 Claudia Emmert: Die Rückkehr der Affekte. Künstlerische Strategien der Affizierung zwischen Inszenierung und Affizierung. In: Claudia Emmert, Jessica Ullrich (Hg.): Affekte, Berlin 2015, S. 20-33, hier: S. 22.

26 Kathrin Busch: Ansteckung und Widerfahrnis. Für eine Ästhetik des Pathischen. In: dies., Iris Därmann (Hg.): „pathos“. Konturen eines kulturwissenschaftlichen Grundbegriffs, Bielefeld 2007, S. 51-73, hier: S. 52.

27 Julia Höner, Kerstin Schankweiler: Affect Me. Social Media Images in Art. In: dies. (s. Anm. 10), S. 13-53, hier: S. 29.

28 Vgl. Rainer Mühlhoff. Theresa Schütz: Die Macht der Immersion. Eine affekttheoretische Perspektive. In: Dawid Kasprowicz, Thiemo Breyer (Hg.): Immersion. Grenzen und Metaphorik des digitalen Subjekts, Jg. 19, 2019, Heft 1, S. 17-35, hier: S. 27. 


\section{Bildkritische Einhegungsversuche}

„Horror-Video - wie in einem Ego-Shooter “. ${ }^{29} \mathrm{Zwar}$ schien die spezifische Ästhetik des Videos in der Berichterstattung oft titelgebend zu sein, doch ging man über assoziative Vergleiche zu Gewaltspielen kaum hinaus. Diese bezogen sich vor allem auf die First-Person-Perspektive, als blickten wir aus den Augen der Person, deren Blick- mit dem Bildfeld kongruiert. Den menschlichen Augensinn simulierend, ist der Körper des Avatars dabei nur fragmentarisch zu sehen - meist in Form der Unterarme und Hände, die eine Waffe halten. An dieser scheinbar unverhandelbaren „Stelle der [...] Immersion ${ }^{\text {“30 }}$ wähnen wir uns mitten im Geschehen. ${ }^{31}$

Werden unter Immersion historisch ganz verschiedene Verfahren der Sichtbarmachung oder des Eintauchens subsummiert, so scheint, wie Dawid Kasprowicz und Thiemo Breyer postulieren, seit den 1990er-Jahren vor allem die Bedeutung einer spektakulären, irritierenden Sinneserfahrung hervorgehoben zu werden, bei der Immersion auch eine „besondere Erwartungshaltung einer involvierenden und Aufmerksamkeit fesselnden Spiel- oder Filmwelt“ adressiert. ${ }^{32}$ Scheint vor allem mit der „Schöpfung neuer virtueller oder fiktionaler Welten ein kognitiv wie auch leiblich umfassend affizierendes Erlebnis“"verbunden zu sein, bietet sich insbesondere für die Untersuchung des Terrorvideos mit Ego-Perspektive an, das Phänomen der Immersion affekttheoretisch anzubinden. ${ }^{33}$

Zunächst verweist die Perspektive auf Körper-Kamera-Hybride, die die Augen des Avatars - als Identifikationsfigur aller Betrachter*innen im virtuellen Raum - zu Bildaufzeichnungs- und Bildausgabemedien machen. Hier entfaltet sich als Effekt der mutmaßlichen „Übereinstimmung von Kameraobjektiv und Betrachterauge“ das affektstarke „Suggestionspotenzial“ des Videos, das Distanzen zwischen Bild und Betrachter*innen tilgt und sich mit der spezifischen Illusionsästhetik des Terrorvideos verbindet. ${ }^{34}$ Im Zusammenhang von Illusion in konzeptueller Funktion einer audiovi-

29 Horror-Video - wie in einem Ego-Shooter, 15.03.19, 20 Minuten, https://www.20min.ch/story/horrorvideo-wie-in-einem-ego-shooter-132090875862 (Stand 4/2020).

30 Rolf Nohr: I've seen the devil of violence. Verhandlungen über das Gewaltmonopol in der ,Killerspieldebatte'. In: AugenBlick. Marburger Hefte zur Medienwissenschaft, 2010, Heft 46: Killerspiele. Beiträge zur Ästhetik virtueller Gewalt, S. 5-17, hier: S. 10.

31 Vgl. Patrick Rupert-Kruse: Im Sog des Blicks. Die Erste-Person-Perspektive als immersive Strategie des Films. In: Jahrbuch für immersive Medien, Jg. 3, 2011, S.37-50, hier: S. 37f.

32 Dawid Kasprowicz, Thiemo Breyer: Immersion oder Das Versprechen der Auflösung. In: dies. (Hg.): Navigationen. Zeitschrift für Medien- und Kulturwissenschaften, Jg. 19, 2019, Heft 1, S. 7-17, hier: S. 7.

33 Ebd.

34 Oliver Grau: Immersion \& Emotion. Zwei bildwissenschaftliche Schlüsselbegriffe. In: ders., Andreas Keil (Hg.): Mediale Emotionen. Zur Lenkung von Gefühlen durch Bild und Sound, Frankfurt a. M. 2005, S. 70-107, hier: S. 80. 
suellen „Realitätssuggestion “35 und Immersion als ein effektvolles Einbezogensein in diese suggerierte Realität ließen sich die technischen Gewaltbilder von Christchurch nicht nur dann als immersiv charakterisieren, wenn „die ikonische Differenz zwischen Bildträger und Bildobjekt nicht mehr wahrgenommen werden kann“. ${ }^{36}$ Ebenso wenig ist „das Versprechen seiner, irgendwie gearteten Echtheit“" zwangsläufig an die Qualität gebunden, sich der „technischen Gemachtheit zu entziehen “, ${ }^{37}$ im Gegenteil: Diese Sicht verfehlt die Spezifik von Body-Cam-Aufzeichnungen in Konfliktsituationen, sich als Medien gerade über das Anzeigen ihrer Medialität in Verwackelung und Unschärfe durch heftige Körperbewegungen selbst auszublenden.

Die Verwackelung ist nicht Zeichen der Künstlichkeit dessen, was die Kamera zu sehen gibt, sondern resultiert aus der physischen Bewegung als Indiz der affektiven Bewegtheit der filmenden Person, die wiederum die akute Konfliktsituation anzeigt und die Betrachter*innen affiziert. Die illusionäre Brechung erklärt sich aus der Handlung selbst und entfaltet so ein hohes (Selbst-)Evidenzpotenzial der Bilder. Die bewegende Drastik des Dargestellten wirkt zusammen mit der drastischen Bewegung der Darstellung, sodass Affekt- und Wirklichkeitspotenzial sich ebenso annähern wie mediale Realität und Lebenswirklichkeit. Das scheint die ,Verwackelungsästhetik' von Body-Cam- und auch vielen Smartphone-Videos von Augenzeug*innen in Konfliktsituationen so attraktiv für Spiel- und Filmfiktionen sowie für sozial- und massenmediale Kommunikations- und Berichterstattungsformate zu machen.

In Kombination mit der bewegt-bewegenden Körper-Ästhetik verwackelter Aufnahmen können der Bildausschnitt mit Unterarm und Waffe und die First-PersonPerspektive im Terrorvideo und Ego-Shooter kurzzeitig verwirren, sodass sich mit der erschwerten „Unterscheidung von ,Spiel, als ob es Ernst wäre“ und ,Ernst, als ob es Spiel wäre“" Grenzen zwischen Fakt und Fiktion scheinbar verschieben. ${ }^{38}$ Doch an der Besonderheit von Ego-Shootern innerhalb bildtheoretischer ComputergameDiskurse lässt sich die Grenze dieser Verwirrung sowie deren ethische und politische Implikationen aufzeigen.

35 Sebastian Lederle: Illusionsästhetik und leibgebundene Immersion im Kinofilm. Anmerkungen zu einer begrifflichen Konstellation. In: Kasprowicz, Breyer (s. Anm. 32), S. 123-143.

36 Stephan Günzel: Simulation und Perspektive. Der bildtheoretische Ansatz in der Computerspielforschung. In: Matthias Bopp, Rolf F. Nohr, Serjoscha Wiemer (Hg.): Shooter. Eine multidisziplinäre Einführung, Münster 2009, S. 331-353, hier: S. 338.

37 Rolf F. Nohr: Das Verschwinden der Maschinen. Vorüberlegungen zu einer Transparenz-Theorie des Games. In: Britta Neitzel, Matthias Bopp, Rolf F. Nohr (Hg.): „See? I'm real ...“ Multidisziplinäre Zugänge zum Computerspiel am Beispiel von ,Silent Hill‘, Münster 2010, S. 96-125, hier: S. 101.

38 Henning Mayer: Soziale Vexierbilder. Zur motivierenden Verklammerung von Spiel und Ernst in virtuellen Beobachtungsarenen. In: Image, 2020, Nr. 31, S. 50-71, hier: S. 59. 
Bei „keinem anderen Spielgenre leitet sich das Spielprinzip derart unmittelbar aus der Bildlichkeit ab “. ${ }^{39}$ Begünstigt die perspektivisch erzeugte und affektiv wirksame Nähe eine Identifikation von Nutzer*in und Spielfigur, so wird dieser Annäherungsprozess problematisch, sobald wir handlungsbezogene und ideologische Konflikte beim Betrachten des Videos bemerken. Bei der Point-of-View-Perspektive als Überblendung von uns, Avatar und Täter ist View räumlich und auch als Referenz auf die eigene Sichtweise zu verstehen, die konstitutiv mit Handlungsoptionen im Spiel verschaltet ist. Im Ego-Shooter ist der Point-of-View als Ort der Handlungsaufforderung und -möglichkeit ein Point-of-Action. ${ }^{40}$

Dieses Phantasma fiktionaler Spiele, in Gewalthandlungen selbst eingreifen zu können, entlarvt und invertiert das Terrorvideo. Wir führen die Morde weder aus noch können wir sie verhindern. Solange wir hinsehen, wird das Grauen andauern und uns an der Schwelle von Bild- und Betrachtungsrealität durch die Sichtbarkeit der Arme, die das Gewehr als apparative Verlängerung unserer Körper in der perspektivischen und affektiven Übergehung der virtuellen Grenze bedienen, buchstäblich im Bild halten. Wir komplettieren imaginativ nicht nur Schnittszenen wie im Clip der Bildzeitung, sondern wir ergänzen auch den fragmentarischen Ego-Leib im Video. Der Täter demonstriert nicht nur seine Macht, indem er auf grausame Weise Menschen massenhaft ermordet, sondern tritt zudem als mächtiger Medienakteur auf, der Blickregime asymmetrisiert, indem er uns durch die Verbindung von Technik und Körper räumlich, affektiv und ideologisch einzuspannen versucht.

Doch die „Bereitschaft, mitunter sogar eine Sehnsucht, sich außeralltäglichen, grenzüberschreitenden und zum Teil vereinnahmenden Erfahrungen hinzugeben“, 41 die sich hinter dem vielfachen Sichtbarwerden von Terrorbildern vermuten lässt, kann „weder von einer anhaltenden Begeisterung für illusionäre Medien hinlänglich erklärt werden, noch lässt sich ein Subjekt voraussetzen, dem es jedes Mal um eine ästhetische Erfahrung samt Reflexionsakt ginge“. ${ }^{42}$ Dass das dynamische Wirkungsgeschehen zwischen Terrorvideo und Betrachter*in auch ,unreflektiert und unterhalb diskursiver Thematisierungen ${ }^{643}$ verlaufen kann, wenn die Betrachter*innen im Moment des Affiziertseins „nicht über Mittel und Kräfte der Distanzierung ${ }^{\text {‘‘4 }}$ zu verfügen

39 Günzel (s. Anm. 36), S. 335.

40 Vgl. Stephan Günzel: Vom Sehen des Sehens zum Sehen des sich selbst Sehens. In: Arno Böhler, Christian Herzog, Alice Pechriggl (Hg.): Korporale Performanz. Zur bedeutungsgenerierenden Dimension des Leibes, Bielefeld 2013, S. 123-157, hier: S. 125 f.

41 Mühlhoff, Schütz (s. Anm. 28), S. 18.

42 Kasprowicz, Breyer (s. Anm. 32), S. 8.

43 Mühlhoff, Schütz (s. Anm. 28), S. 18.

44 Ebd., S. 30. 
scheinen, mag ebenso ein Attraktionsfaktor sein. Um aber herauszuarbeiten, ,wie das Individuum“ in diese „Dynamik selbst affizierend eingebunden ist“ und inwiefern sich in diesem Fall Immersion „als ein bestimmter Typus affektiver Einbindung “45 auszeichnet, sind reflexive Distanzierungsangebote notwendig.

So wird eine Zusammenführung ethisch-politischer Kontextualisierungen und bildkritischer Einhegungen für den sozial- und massenmedialen Umgang mit technischen Gewaltbildern von Aufmerksamkeitsverbrechen wichtig - zum einen, um deren Anziehungskraft mehrperspektivisch einzuordnen, die sich insbesondere aus dem Wechselverhältnis der Angst vor und des Begehrens nach einer Überwältigung durch affektstarke Bilder zu speisen scheint, und zum anderen, um an aktuelle Diskussionen über mögliche Zensurmaßnahmen anzuschließen.

\section{Ausblick}

Nachdem die EU-Kommission schon ein Jahr vor dem Terror in Christchurch soziale Plattformen um eine „freiwillige“ Zensur bat, ${ }^{46}$ forderte sie wenige Monate später in einem Verordnungsentwurf, ,von sämtlichen Anbietern von Hosting-Diensten in der EU, terroristische Inhalte innerhalb einer Stunde [...] zu löschen“ “. ${ }^{47}$ Auch durch proaktive Mittel wie Uploadfilter sollten sie deren Verbreitung eindämmen. Mögen gesetzliche Filter- und Löschungsvorgaben dem Schutz der Nutzer*innen dienen, gefährden sie zum Teil dennoch die Meinungs- und Pressefreiheit. Künstliche Intelligenzen können intentionale und ideologische Hintergründe nicht herausfiltern. Wissenschaftliche, sensationsgierige, extremistische oder antiterroristische Nutzungsabsichten geraten potenziell durcheinander. Diese Einordnung ist aber vor allem bei Terror, der sich klaren Definitionen entzieht, unumgänglich.

Anfang April 2019 sollte es vorerst keine gesetzlichen Uploadfilter, aber eine Löschfrist von 24 Stunden geben - eine Frist, in der Facebook die millionenfache Verbreitung des Videos trotz millionenfacher Löschung nicht unterbinden konnte. ${ }^{48}$ Viele Nutzer*innen bearbeiteten das Originalvideo in Schnitt oder Ton. So geringfügig die

45 Ebd., S. 27.

46 Vgl. Tomas Rudl: EU-Kommission will von Plattformen „freiwillige“ und weitreichende Internetzensur, 02.03.18, netzpolitik.org, https://netzpolitik.org/2018/eu-kommission-will-von-plattformen-freiwilligeund-weitreichende-internetzensur/ (Stand 4/2020).

47 Tomas Rudl: Wundermittel Uploadfilter gegen Terrorpropaganda: EU-Mitgliedsstaaten auf Linie der EU-Kommission, 30.10.18, netzpolitik.org, https://netzpolitik.org/2018/wundermittel-uploadfiltergegen-terrorpropaganda-eu-mitgliedstaaten-auf-linie-der-eu-kommission/ (Stand 4/2020).

48 Vgl. Tomas Rudl: EU-Parlamentsausschuss: Keine Uploadfilter gegen den Terror, aber kurze Löschfrist, 08.04.19, netzpolitik.org, https://netzpolitik.org/2019/eu-parlamentsausschuss-keine-uploadfilter-gegenden-terror-aber-kurze-loeschfrist/ (Stand 4/2020). 
Veränderungen ästhetisch zu sein schienen, so entscheidend waren sie für die Effizienz des technischen Erkennungssystems, das die bearbeiteten Versionen nicht mehr dem Originalvideo zuweisen und so deren Upload nicht verhindern konnte. ${ }^{49}$ Ließe sich hier eine ästhetische ,Überempfindlichkeit' vermuten, ginge mit einer ,Vergröberung' der Erkennungsfilter gleichwohl eine ästhetische ,Oberflächlichkeit' einher, die assoziative Kurzschlüsse beförderte und zwei Gefahren mit sich brächte. Erstens könnten im Fall von Christchurch auch viele legale Gameplay-Videos gelöscht werden, sodass Filtergesetze im sogenannten Kampf gegen den Terror nicht zum Blueprint netzpolitischer Einschränkungen werden sollten. ${ }^{50}$ Zweitens könnten Videos mit falschen Informationen verknüpft werden und zu faktischer Desorientierung mit verschwörungsmythischer Anschlussfähigkeit beitragen - so geschehen, als YouTube im April 2019 in Live-Stream-Videos der brennenden Kathedrale Notre Dame Informationsfelder zu 9/11 mit Bildern der brennenden Türme des World Trade Centers integrierte. So scheinen gerade jene zwei besprochenen Praxen - die medienethische und -politische Kontextualisierung der Tatbilder ebenso wie die bildkritische Analyse ihrer affektiven Wirkungskräfte - auch für die Diskussion technischer Umgangsmöglichkeiten mit terroristischen Gewaltbildern von Bedeutung sein zu können.

49 Vgl. Patrick Beuth: Wie sich das Livevideo des Halle-Attentäters verbreiten konnte, 10.10.19, Der Spiegel, https://www.spiegel.de/netzwelt/web/halle-wie-sich-das-taetervideo-auf-twitch-telegram-und-facebookverbreitet-a-1290841.html (Stand 10/2019).

50 Vgl. Julia Reda: Edit-Policy: Uploadfilter mit KI - EU-Pläne bergen große Risiken, 02.03.20, heise online, https://www.heise.de/newsticker/meldung/Edit-Policy-Uploadfilter-mit-KI-EU-Plaene-bergen-grosseRisiken-4672028.html (Stand 5/2020). 